\title{
Bridging the research gap between marketing academics and practitioners
}

Received: 1st June, 2003

\author{
Behram J. Hansotia \\ is Co-founder, President and Chief Executive Officer of InfoWorks. His expertise lies in designing information-intensive \\ marketing processes for customer acquisition, development and win-back. The approach is embodied in InfoWorks' Customer \\ Value Enhancement ${ }^{\mathrm{TM}}$ process. Behram's post-doctoral experience spans academia, industry and management consulting, \\ with the past several years devoted exclusively to strategic database marketing. Behram is an adjunct professor in the \\ graduate Integrated Marketing Communications programme at Northwestern University, has been a member of the Editorial \\ Board of the Journal of Interactive Marketing and currently serves on the International Editorial Board of this journal.
}

\begin{abstract}
This paper argues that the most useful academic research does not get used by practitioners until many years later. Like any innovation, there is a diffusion process before a method proposed by academics becomes mainstream or at least widely accepted by leading practitioners. Key barriers to faster adoption are identified and some solutions suggested. Rapid knowledge diffusion is important to marketing, since it still lags behind many business functions in rapid productivity enhancements. Academics, practitioners, universities, business and government all have a role in creating faster knowledge diffusion. Academics need to make their work more accessible and easy to use. Universities need to recognise their faculty members' contributions, when their work changes accepted practice. Business organisations create the environment and the culture for practitioners, and if the culture fosters risk taking, encourages learning and aligns reward systems for pioneering activities, faster adoption can occur. Finally, government can help facilitate the knowledge transfer process by funding university-based institutes, with the specific charter of creating faster knowledge dissemination.
\end{abstract}

Behram Hansotia InfoWorks, 10 South Riverside Plaza, Suite 1920, Chicago Illinois 60606, USA.

Tel: +1 3125890261 ; Fax: +1 312559 1061; e-mail: bhansotia@ infoworks-chicago.com

\section{INTRODUCTION}

Many academics are prolific writers and regularly publish several articles each year. Since there is an enormous variance in their interests and backgrounds as well as the motivation for their writing, there is also an enormous variance in what is written, how it is written and the ultimate audience for whom it is written. At the same time, marketing practitioners, who ostensibly are also a key audience for some of this writing, come from a wide variety of backgrounds, have different interests and typically have little patience with things they have difficulty understanding, or feel is of little relevance to them.

Much of the writing in the top tier, refereed marketing journals (for example, Marketing Science, Journal of Marketing Research and Journal of Consumer Research) is consumed almost exclusively by academics in major research universities. These journals exist for that very reason: to provide a channel for marketing scientists to get their work critically evaluated by leading scholars and, if it is blessed, to make it available to the 
marketing community of academicians and practitioners.

\section{KNOWLEDGE DIFFUSION}

It is not that new techniques and methods never make it to the world of the practitioner. They do. It takes time but, just as any innovation goes through a diffusion process before it becomes mainstream, the best and most useful techniques do eventually get accepted and used by practitioners. Examples are conjoint measurement and analysis, response modelling and segmentation. This is because within the practitioner community there are a few immigrants from academia, who still keep up with the literature and are quite interested in applying new methods to their problems. These ex-academics are either consultants or work within specialised departments of corporations. Here is an example.

In 1972, David Cox ${ }^{1,2}$ published his seminal paper on hazard regression. (Hazard regression deals with predicting/explaining the timing of events.) Over the next two decades hundreds of articles appeared on this topic mainly in statistical journals and even a few books were published in this area. Several of the applied articles were in the field of biostatistics and sociology, where the interest was on the timing of events such as a patient's death/cure, or the recidivism rate. It was not until the early 1990s, however, when Jain and Vilcassim $^{3}$ published an article on the timing of coffee purchases, that researchers in the marketing community started getting interested in timing issues. At about the same time, Paul Allison, ${ }^{4}$ a sociologist at the University of Pennsylvania, started offering intensive three to four-day seminars on hazard regression (sociologists call it event history analysis) to industry professionals.
The author attended the seminar in the summer of 1992. Most of the attendees were researchers in the biostatistics area; there were, however, two or three marketing professionals in the class. The author was one of them, he was then a database marketing consultant and had recently worked on a lifetime value (LTV) project for a client who marketed continuity products. The author was intrigued about modelling customer attrition rates and then using the attrition score as one of the dimensions for segmenting customers. Later, in the mid-to-late 1990s, hazard regression was used repeatedly on several client projects. The key reasons this was possible were that the consultancy had:

— the right data

- an understanding of the methodology

- significant expertise with SAS and SAS had the right procedures for the job (LIFETEST, LIFEREG, PHREG, GENMOD).

This is how diffusion occurs. Nearly 25 years had passed from David Cox developing the hazard regression methodology to the consultancy using it, and yet they felt like pioneers when they first applied it successfully and were delighted with the results. The client liked the work a lot, since it did differentiate her customer base into radically different groups (based on LTV) and it also provided insights into the drivers of early attrition. She could not have cared less, however, about how those results were achieved. The most important factor in the adoption of new methods by practitioners is readily available, proven software.

The author's firm went through a similar experience again about five or six years ago, when they adopted latent class modelling as their principle approach to segmenting customers. Latent class 
modelling posits that the observed data hail from a mixture of probability distributions and the method untangles this mixture so the probabilities of a customer belonging to each of the underlying distributions (segments) can be estimated. One of the young recruits that year had a PhD from the University of Chicago and had been exposed to latent class modelling, and although SAS did not have a procedure for this method (and still does not have one) Professor Vermunt, at Tilburg University in the Netherlands, had posted his $\ell_{\mathrm{EM}}$ program on the internet and it was readily available. Had he not, the firm might still be using SAS's K-means procedure (FASTCLUS) to segment customers. (There is nothing wrong with $\mathrm{K}$-means but it does not easily lend itself to handling categorical variables.)

The most useful research does end up being used by practitioners, however it takes time. Several factors can shorten this diffusion time. First, however, this paper discusses how this diffusion process occurs in marketing.

\section{MARKETING ROOTS AND DIFFUSION OF MARKETING KNOWLEDGE}

Several of the most important approaches to key marketing problems have their origins in psychology, statistics and economics. Many leading marketing scholars subscribe to the most advanced journals in these fields and often have dual interests. The methodologies discussed in these journals influence their own research but in the context of marketing issues. This results in working papers and presentations in conferences and eventual publication in one of the top tier research-oriented marketing journals. Once this occurs other marketing researchers follow this thread of work. They generalise the assumptions, introduce refinements in the methodology and sometimes develop innovative extensions to the approach. Often, new computer programs are written but they are generally not widely disseminated or posted on websites.

These same professors now start requiring their $\mathrm{PhD}$ students to read these articles and some of their students write dissertations on extensions of these methodologies. At the same time other marketing academics may develop edited books of readings as well as graduate-level textbooks. The latter obviously takes some time. In the meantime, practitioners may also have read some of these articles and books. But the actual application of these methods does not occur unless it is relatively painless to apply them. Only when reasonably easy-to-use computer programs are readily accessible, and in the public domain, will we see increased usage of the academic research, even by the most sophisticated practitioners. Figure 1 depicts the diffusion process through a flow diagram. Much more, however, needs to occur before some of these methodologies become mainstream. Even today, many large companies who do a significant amount of direct marketing do not systematically use targeting tools, eg regression models, to make their campaigns more efficient. The effectiveness of regression models has been well established and they would hardly be considered cutting-edge tools, so obviously there is a fundamental problem.

\section{BARRIERS TO FASTER ADOPTION OF MARKETING RESEARCH}

The barriers to faster adoption of the research are:

- cultural

- organisational 


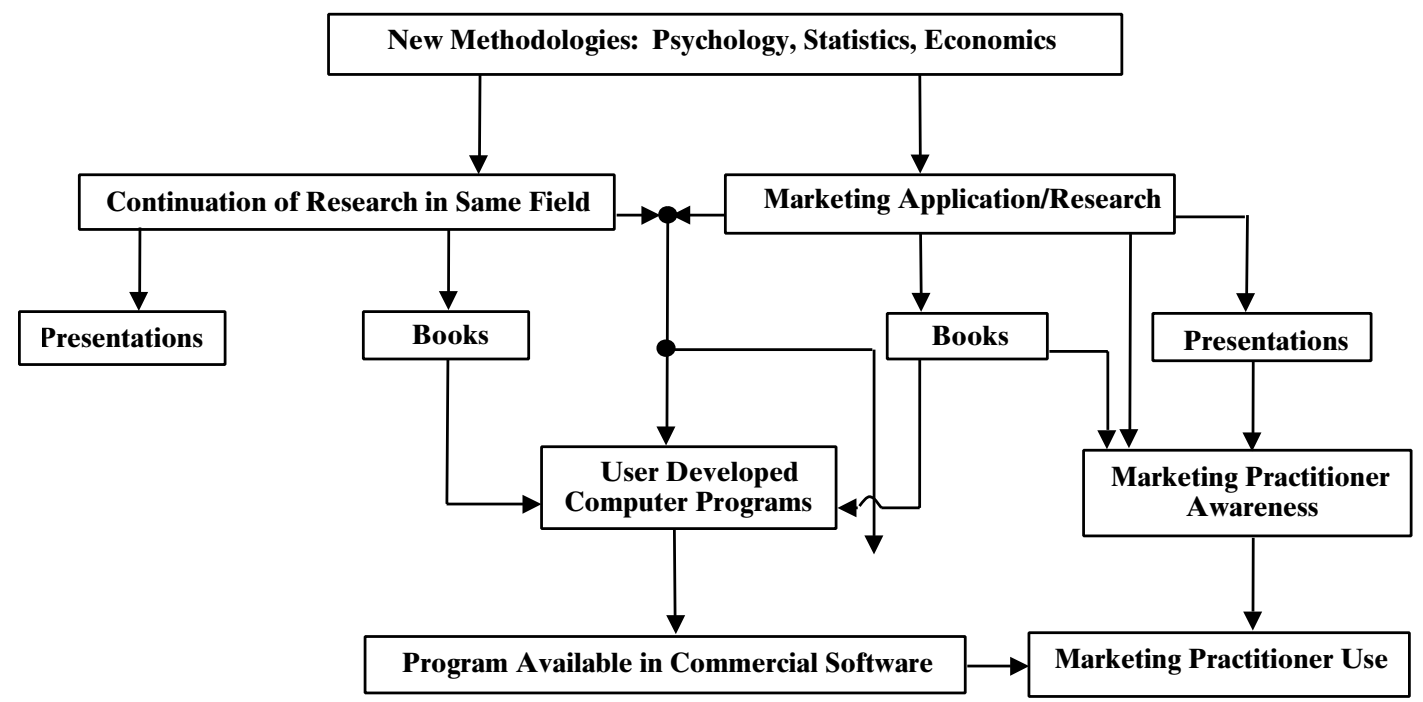

Figure 1: Flow diagram of diffusion process for marketing innovations/research

- lack of proof of superiority of new approach

- lack of rewards for faster adoption of new methods

- awareness

- training/education

- lack of availability of specialised programs that are easy to use.

Each of these barriers is discussed next.

\section{Cultural barriers}

How aggressively a company adopts new methods is largely a function of the company's culture and its willingness to take risks in the pursuit of superior performance. To a large extent, this is influenced by the company's history and the type of organisation the CEO and the senior executives wish to create. An organisation with a learning culture where employees are not punished for trying new things provides considerable freedom in how employees approach different problems and attracts individuals who want to continue to grow and develop. Also, an organisation that believes in the development of 'core competencies' as a major strategy is more likely to encourage specialisation in key disciplines and this facilitates faster adoption of new methodologies. Changing a moribund culture is monumentally difficult and can never be achieved unless there is a total commitment by the CEO and the team in creating the change. The rewards however can be significant, both for employees and shareholders.

\section{Organisational barriers}

Certain businesses are ideally suited for database and direct marketing, yet a number of these companies continue to use primarily brand advertising and/or a sales force. The database and direct marketing disciplines are not deemed important enough for these companies to invest in specialists in these disciplines. Other companies that do make extensive use of direct marketing often staff these positions with generalists and outsource the database and analytics to agencies and consultants. For academic research to be used more extensively, companies need to hire more specialists with advanced 
degrees and even pursue (applied) R\&D in marketing science to improve their competency in the technical areas of marketing (where a lot of the academic research is taking place).

\section{Lack of proof of superiority of new approach}

Very few research articles document the financial impact of using a new, more advanced technique rather than one in current use by practitioners. For example, a newer method may explicitly take into consideration sources of additional variation (customer heterogeneity) using random effects or, explicitly, account for the auto-correlation in repeated observations in regression models. Such approaches do result in better estimates of model parameters and more accurate tests of hypotheses; however, academics seldom document the financial impact and opportunity cost of using a less accurate model.

If a new method can be shown to reveal new insights, or to produce superior forecasts or to create improved discrimination that result in substantially improved financial performance, the new methods will be adopted faster.

\section{Lack of rewards for faster adoption of new methods}

Faculty members get both psychological and financial rewards if their research gets cited extensively by other researchers. It implies a significant contribution to the field. There is, however, no similar recognition or reward for the research being used extensively by practitioners. If major corporations, the potential beneficiaries of the research, created an annual reward for a methodology that helped change how companies address critical marketing problems, it would motivate academic researchers to help facilitate this change. This would influence how academics demonstrate the value of their approach, how they would write their papers (so they are more accessible to practitioners) and where they publish their work.

\section{Awareness barriers}

Unless practitioners have received significant advanced training/education, or have academic roots, they typically do not subscribe to the refereed journals where the most advanced, academic research is published. It behoves researchers to take their findings and approaches and repackage them for a less technical audience. There are very few journals dedicated to this activity. Publications like Harvard Business Review do have articles, from time to time, where professors present the implications of their work so it appeals to senior executives. There are, however, no journals geared to practitioners that explain new methodologies with a minimum of mathematics, explain the business benefits in practical terms and lay out step-by-step how the new methodologies should be used.

\section{Training/education barriers}

A lot of training and educational opportunities do exist, but mostly they address well-established approaches that were developed many years ago. The educational recipients of the latest research are typically other professors and doctoral students in advanced seminars in research universities. These seminars would not work for practitioners. The objective of both the seminars and the journals is to disseminate the new research to a group well versed in the more recent, advanced methodologies, implicitly assuming significant prior 
knowledge of previous research in the specific field.

Special courses need to be tailored to practitioners' backgrounds and needs. These courses should address specific problems important to industry, which current training methods are not addressing adequately. As far as possible, the courses should clearly establish the value of the new approach in comparison to the old, with value being measured in financial terms or ease of use. This could be a powerful motivator for adoption of a new method.

(When conjoint analysis was first developed, researchers recommended using linear programming and robust regression methods to estimate the part-worths of the decision makers' value functions. Later it was shown that even though multiple linear regression (MLR) violated some of the underlying assumptions the results were not markedly different. Since MLR programs were/are extensively available, it became the de facto approach to carrying out conjoint analyses.)

\section{Lack of availability of computer programs}

This may be the single most important barrier preventing faster adoption of new approaches by leading consultants and companies. If academics posted their computer programs, with instructions on using them on the web, more non-academics would start using them. Since a high percentage of this work is funded by non-profit organisations, namely universities and governments, giving tax-paying organisations ready access to these programs would definitely help disseminate knowledge faster and improve the performance of organisations availing themselves of this opportunity.

To facilitate this dissemination, a university-based international institute could be set up to invite academics to submit their programs (with supporting documentation) for posting at the institute's website. The website could provide quarterly reports of the downloads to the authors of the programs and this could be used as an additional metric in evaluating and rewarding academics. To facilitate even greater use, the institute could set up some minimal requirements for posting the programs. For instance:

- program documentation

- examples of program applications

- discussion of output

- preferred programming language (SAS, IML, C ++ , etc)

- author contacts, etc.

There should also be some requirements placed on individuals downloading these programs. For instance, they may not sell the programs 'as is' to another party; they may not be enhanced and then sold without the prior consent of the authors, etc. In other words, there should be some protection of the intellectual content, for the authors. Obviously, several additional details need to be worked out before an idea like this becomes reality.

\section{SUMMARY}

A huge volume of material written by faculty members is published in a variety of magazines and trade and professional journals. Research universities encourage this publication and their prestige is directly gauged by the number of articles their faculty publish in top tier, refereed journals. The gates to these journals are well guarded by a distinguished editor and editorial board as well as referees who are friends and colleagues of the editorial board and have themselves published in these journals before. 
Arguably, the most advanced knowledge created is published in refereed journals and yet there is this uneasy feeling that the very audience these articles should be influencing the most, ie practitioners, is either blissfully unaware of the work or chooses to ignore it.

This paper suggests that if research is truly useful it is eventually used by practitioners. In most instances the process is painfully slow and often takes decades before companies start experimenting. Seven barriers to more rapid diffusion of the knowledge base are identified in this paper and remedies to address them proposed. Some of the challenges, like organisational culture, are quite formidable and can only be addressed through a painful change process by a determined CEO and the senior team. Other barriers could be addressed with financial assistance from corporations and governments. Discussing this important problem in a journal read by both academicians and practitioners is, however, the first step in creating a dialogue between several parties and institutions that have a stake in knowledge dissemination.

\section{References}

1 Cox, D. R. (1972) 'Regression models and life tables', Journal of the Royal Statistical Society, B34, pp. 187-220.

2 Cox, D. R. and Oakes, D. (1984) 'Analysis of survival data', Chapman \& Hall, London.

3 Jain, D. C. and Vilcassim, N. J. (1991) 'Investigating household purchase timing decisions: A conditional hazard function approach', Marketing Science, Vol. 10, pp. 1-23.

4 Allison, P. D. (1984) 'Event history analysis', Sage Publications, Beverly Hills. 